\title{
Correction to: Spatial navigation in early multiple sclerosis: a neglected cognitive marker of the disease?
}

\author{
Eva Němá ${ }^{1}$ Adam Kalina ${ }^{1} \cdot$ Tomáš Nikolai $^{1} \cdot$ Martin Vyhnálek $^{1} \cdot$ Eva Meluzínová $^{1} \cdot$ Jan Laczó $^{1}$
}

Published online: 30 December 2020

○) Springer-Verlag GmbH Germany, part of Springer Nature 2020

\section{Correction to: Journal of Neurology} https://doi.org/10.1007/s00415-020-10079-z

The original version of this article unfortunately contained a mistake. The correct information is given below.

The given names and family names were interchanged.

The names should be:

Eva Němá · Adam Kalina · Tomáš Nikolai · Martin Vyhnálek · Eva Meluzínová · Jan Laczó

Affiliation should be:

Department of Neurology, 2nd Faculty of Medicine, Charles University and Motol University Hospital, V Uvalu 84, 15006 Prague, Czech Republic

Figure 1 caption should read:

Fig. 1 Human analogue of the Morris Water Maze task. a The real-space navigation setting. bThe scheme of the task showing an aerial view of the arena (large white circle) with starting point (red filled circle), orientation cues (red and green lines), and goal (purple circle).

Figure 2 caption should read:

Fig. 2 Voxels with significant $(p<0.05)$ grey matter volume reduction in the early MS group compared to controls in rendered brains projected to show: A) left side, B) right side, C) front, D) back, E) top and F) bottom.

Figure 3 caption should read:

Fig. 3 Voxels with significant $(p<0.05)$ grey matter volume reduction in controls compared to the early MS group in rendered brains projected to show: A) left side, B) right side, C) front, D) back, E) top and F) bottom.

Table 1 should be:

The original article can be found online at https://doi.org/10.1007/ s00415-020-10079-z.

Jan Laczó

jan.laczo@1fmotol.cuni.cz

1 Department of Neurology, 2nd Faculty of Medicine, Charles University and Motol University Hospital, V Úvalu 84, 15006 Prague, Czech Republic 
Table 1 Characteristics of the study participants

\begin{tabular}{|c|c|c|c|c|}
\hline & Controls $(n=41)$ & Early MS $(n=52)$ & $p$ values & Effect sizes \\
\hline \multicolumn{5}{|l|}{ Demographic characteristics } \\
\hline Age (years) & $29.02(7.50)$ & $31.29(6.98)$ & 0.135 & $0.32^{\mathrm{a}}$ \\
\hline Women, $n(\%)$ & $23(54.80)$ & $28(54.90)$ & 0.989 & $0.00^{\mathrm{b}}$ \\
\hline Education (years) & $16.02(2.58)$ & $15.06(2.87)$ & 0.095 & $0.35^{\mathrm{a}}$ \\
\hline EDSS (score) & NA & $1.67(0.58)$ & NA & NA \\
\hline \multicolumn{5}{|l|}{ Neuropsychological characteristics ${ }^{c}$} \\
\hline BDI (score) & $4.74(4.94)$ & $8.49(10.17)$ & 0.047 & $0.05^{\mathrm{d}}$ \\
\hline BAI (score) & $5.14(5.07)$ & $9.67(10.07)$ & 0.013 & $0.08^{\mathrm{d}}$ \\
\hline MSNQ (score) & $15.98(11.36)$ & $13.71(10.02)$ & 0.747 & $0.00^{\mathrm{d}}$ \\
\hline NART (score) & $118.60(18.88)$ & $116.06(8.78)$ & 0.490 & $0.01^{\mathrm{d}}$ \\
\hline RAVLT 1-5 (score) & $58.74(7.69)$ & $52.51(9.29)$ & 0.003 & $0.10^{d}$ \\
\hline RAVLT delayed (score) & $12.60(2.06)$ & $10.67(2.78)$ & 0.002 & $0.11^{\mathrm{d}}$ \\
\hline BVMT-R 1-3 (score) & $30.31(4.00)$ & $26.41(4.80)$ & $<0.001$ & $0.16^{\mathrm{d}}$ \\
\hline BVMT-R delayed (score) & $11.10(1.12)$ & $10.24(1.62)$ & 0.009 & $0.08^{\mathrm{d}}$ \\
\hline SDMT (score) & $64.29(11.17)$ & $56.14(10.52)$ & 0.004 & $0.10^{d}$ \\
\hline PASAT (score) & $51.57(7.23)$ & $44.80(11.32)$ & 0.003 & $0.10^{d}$ \\
\hline Digit span forward (score) & $10.24(2.01)$ & $8.98(2.00)$ & 0.007 & $0.09^{\mathrm{d}}$ \\
\hline Digit span backward (score) & $8.00(2.93)$ & $6.92(2.40)$ & 0.099 & $0.03^{\mathrm{d}}$ \\
\hline TMT A (time in s) & $27.40(20.92)$ & $31.92(9.22)$ & 0.208 & $0.02^{\mathrm{d}}$ \\
\hline TMT B (time in s) & $53.07(13.30)$ & $71.37(25.43)$ & $<0.001$ & $0.16^{\mathrm{d}}$ \\
\hline COWAT (score) & $48.67(13.60)$ & $39.33(11.43)$ & 0.001 & $0.13^{d}$ \\
\hline JLO (score) & $27.52(3.39)$ & $25.67(4.42)$ & 0.028 & $0.06^{\mathrm{d}}$ \\
\hline CFT animals (score) & $26.81(5.63)$ & $24.45(6.43)$ & 0.056 & $0.05^{\mathrm{d}}$ \\
\hline CFT shopping (score) & $26.79(8.18)$ & $25.55(7.72)$ & 0.312 & $0.01^{\mathrm{d}}$ \\
\hline \multicolumn{5}{|l|}{ Navigational characteristics ${ }^{c}$} \\
\hline Egocentric navigation (distance, $\mathrm{cm}$ ) & $18.64(7.39)$ & $31.82(27.76)$ & $0.010^{\mathrm{e}}$ & $0.07^{d}$ \\
\hline Allocentric navigation (distance, $\mathrm{cm}$ ) & $22.60(8.22)$ & $38.21(23.69)$ & $0.004^{\mathrm{e}}$ & $0.09^{d}$ \\
\hline Allocentric delayed navigation (distance, $\mathrm{cm}$ ) & $24.57(12.21)$ & $34.45(27.74)$ & $\mathbf{0 . 0 3 8}^{\mathrm{e}}$ & $\mathbf{0 . 0 5}{ }^{\mathrm{d}}$ \\
\hline \multicolumn{5}{|l|}{ MRI volumetric characteristics ${ }^{\mathrm{c}}$} \\
\hline $\mathrm{nBP}\left(\right.$ volume, $\mathrm{cm}^{3}$ ) & $1507.75(49.64)^{\mathrm{f}}$ & $1464.89(71.09)$ & 0.005 & $0.10^{\mathrm{d}}$ \\
\hline nWM (volume, $\mathrm{cm}^{3}$ ) & $703.40(34.09)^{\mathrm{f}}$ & $677.87(38.06)$ & 0.007 & $0.10^{\mathrm{d}}$ \\
\hline nGM (volume, $\mathrm{cm}^{3}$ ) & $804.34(35.64)^{\mathrm{f}}$ & $787.01(53.31)$ & 0.064 & $0.05^{\mathrm{d}}$ \\
\hline npGM (volume, $\mathrm{cm}^{3}$ ) & $638.76(33.02)^{\mathrm{f}}$ & $618.16(42.36)$ & 0.009 & $0.09^{\mathrm{d}}$ \\
\hline Amygdala left (volume, $\mathrm{mm}^{3}$ ) & $1668.41(249.56)^{\mathrm{f}}$ & $1426.00(236.57)$ & $<0.001$ & $0.18^{d}$ \\
\hline Amygdala right (volume, $\mathrm{mm}^{3}$ ) & $1447.44(213.50)^{\mathrm{f}}$ & $1505.38(270.33)$ & 0.127 & $0.03^{\mathrm{d}}$ \\
\hline Caudate nucleus left (volume, $\mathrm{mm}^{3}$ ) & $3689.21(401.20)^{\mathrm{f}}$ & $3427.88(519.02)$ & 0.041 & $0.06^{\mathrm{d}}$ \\
\hline Caudate nucleus right (volume, $\mathrm{mm}^{3}$ ) & $3590.62(498.15)^{\mathrm{f}}$ & $3595.89(565.43)$ & 0.687 & $0.00^{\mathrm{d}}$ \\
\hline Hippocampus left (volume, $\mathrm{mm}^{3}$ ) & $4146.49(550.70)^{\mathrm{f}}$ & $3851.45(584.50)$ & 0.025 & $0.07^{\mathrm{d}}$ \\
\hline Hippocampus right (volume, $\mathrm{mm}^{3}$ ) & $4174.19(488.38)^{\mathrm{f}}$ & $3931.15(562.88)$ & 0.085 & $0.04^{\mathrm{d}}$ \\
\hline Globus pallidus left (volume, $\mathrm{mm}^{3}$ ) & $1937.87(226.96)^{\mathrm{f}}$ & $1816.70(231.26)$ & 0.037 & $0.06^{\mathrm{d}}$ \\
\hline Globus pallidus right (volume, $\mathrm{mm}^{3}$ ) & $1972.83(275.16)^{\mathrm{f}}$ & $1854.15(239.10)$ & 0.066 & $0.05^{\mathrm{d}}$ \\
\hline Putamen left (volume, $\mathrm{mm}^{3}$ ) & $5367.57(748.49)^{\mathrm{f}}$ & $5053.67(633.66)$ & 0.052 & $0.05^{\mathrm{d}}$ \\
\hline Putamen right (volume, $\mathrm{mm}^{3}$ ) & $5223.60(810.85)^{\mathrm{f}}$ & $5071.32(673.14)$ & 0.480 & $0.01^{\mathrm{d}}$ \\
\hline Thalamus left (volume, $\mathrm{mm}^{3}$ ) & $8831.50(1090.09)^{\mathrm{f}}$ & $7998.49(918.22)$ & $<0.001$ & $0.17^{d}$ \\
\hline Thalamus right (volume, $\mathrm{mm}^{3}$ ) & $8401.30(1052.73)^{\mathrm{f}}$ & $7777.74(948.27)$ & 0.012 & $0.08^{\mathrm{d}}$ \\
\hline \multicolumn{5}{|l|}{ Global and regional MRI lesion load characteristics } \\
\hline Total lesion load (volume, $\mathrm{mm}^{3}$ ) & NA & $3363.22(5328.87)$ & NA & NA \\
\hline Caudate nucleus left (volume, $\mathrm{mm}^{3}$ ) & NA & $150.69(190.58)$ & NA & NA \\
\hline Caudate nucleus right (volume, $\mathrm{mm}^{3}$ ) & NA & $140.51(183.85)$ & NA & NA \\
\hline Cerebellum left (volume, $\mathrm{mm}^{3}$ ) & NA & $10.96(35.12)$ & NA & NA \\
\hline
\end{tabular}


Table 1 (continued)

\begin{tabular}{|c|c|c|c|c|}
\hline & Controls $(n=41)$ & Early MS $(n=52)$ & $p$ values & Effect sizes \\
\hline Cerebellum right (volume, $\mathrm{mm}^{3}$ ) & NA & $9.49(28.41)$ & NA & NA \\
\hline Frontal lobe left (volume, $\mathrm{mm}^{3}$ ) & NA & $104.90(265.65)$ & NA & NA \\
\hline Frontal lobe right (volume, $\mathrm{mm}^{3}$ ) & NA & $116.22(258.26)$ & NA & NA \\
\hline Insula left (volume, $\mathrm{mm}^{3}$ ) & NA & $3.09(10.51)$ & NA & NA \\
\hline Insula right (volume, $\mathrm{mm}^{3}$ ) & NA & $15.49(77.66)$ & NA & NA \\
\hline Occipital lobe left (volume, $\mathrm{mm}^{3}$ ) & NA & $265.35(410.59)$ & NA & NA \\
\hline Occipital lobe right (volume, $\mathrm{mm}^{3}$ ) & NA & $156.92(268.15)$ & NA & NA \\
\hline Parietal lobe left (volume, $\mathrm{mm}^{3}$ ) & NA & $230.02(441.07)$ & NA & NA \\
\hline Parietal lobe right (volume, $\mathrm{mm}^{3}$ ) & NA & $277.51(553.62)$ & NA & NA \\
\hline Putamen left (volume, $\mathrm{mm}^{3}$ ) & NA & $2.98(11.64)$ & NA & NA \\
\hline Putamen right (volume, $\mathrm{mm}^{3}$ ) & NA & $1.43(3.83)$ & NA & NA \\
\hline Temporal lobe left (volume, $\mathrm{mm}^{3}$ ) & NA & $156.90(339.75)$ & NA & NA \\
\hline Temporal lobe right (volume, $\mathrm{mm}^{3}$ ) & NA & $167.02(352.29)$ & NA & NA \\
\hline Thalamus left (volume, $\mathrm{mm}^{3}$ ) & NA & $3.84(13.05)$ & NA & NA \\
\hline Thalamus right (volume, $\mathrm{mm}^{3}$ ) & NA & $4.41(9.85)$ & NA & NA \\
\hline Periventricular left (volume, $\mathrm{mm}^{3}$ ) & NA & $678.12(1315.60)$ & NA & NA \\
\hline Periventricular right (volume, $\mathrm{mm}^{3}$ ) & NA & $865.20(1466.74)$ & NA & NA \\
\hline
\end{tabular}

Demographic, neuropsychological, navigational and MRI characteristics. The values represent mean (SD) unless indicated otherwise. Values in bold indicate significant differences after Holm-Bonferroni correction for multiple comparisons

$M S$ multiple sclerosis, EDSS Expanded Disability Status Scale, BDI Beck Depression Inventory, BAI Beck Anxiety Inventory, MSNQ Multiple Sclerosis Neuropsychological Questionnaire, NART National Adult Reading Test, RAVLT Rey Auditory Verbal Learning Test, RAVLT delayed RAVLT delayed recall after 30 min, BVMT-R Brief Visuo-spatial Memory Test Revised, BVMT-R delayed BVMT-R delayed recall after 25 min, SDMT Symbol Digit Modalities Test, PASAT Paced Auditory Serial Addition Test, TMT Trail Making Test, COWAT Controlled Oral Word Association Test, $J L O$ Judgement of Line Orientation, $C F T$ Category Fluency Test, $n B P$ normalized brain parenchymal volume, $n W M$ normalized white matter volume, $n G M$ normalized grey matter volume, $n p G M$ normalized peripheral grey matter volume

${ }^{a}$ Effect size reported using Cohen's $d$

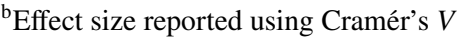

${ }^{c}$ Based on the analyses controlled for age, gender and education

${ }^{\mathrm{d}}$ Effect size reported using partial eta-squared

${ }^{\mathrm{e}}$ Based on an analysis of covariance with log transformed data

${ }^{\mathrm{f}}$ Based on a sample restricted to those who underwent an experimental MRI protocol $(n=32)$

Table 4 should be: 
Table 4 Characteristics of the participants with early multiple sclerosis with normal and less accurate spatial navigation

Normal navigators $(n=37) \quad$ Less accurate navigators $\quad p$ value

Effect sizes $(n=14)$

\begin{tabular}{|c|c|c|c|c|}
\hline \multicolumn{5}{|l|}{ Demographic characteristics } \\
\hline Age (years) & $31.81(6.28)$ & $29.93(8.68)$ & 0.395 & $0.27^{\mathrm{a}}$ \\
\hline Women, $n(\%)$ & $21(56.80)$ & $7(50.00)$ & 0.665 & $0.06^{\mathrm{b}}$ \\
\hline Education (years) & $15.51(2.85)$ & $13.86(2.66)$ & 0.066 & $0.67^{\mathrm{a}}$ \\
\hline EDSS (score) & $1.61(0.50)$ & $1.82(0.75)$ & 0.245 & $0.37^{\mathrm{a}}$ \\
\hline \multicolumn{5}{|l|}{ Neuropsychological characteristics ${ }^{c}$} \\
\hline BDI (score) & $8.95(11.33)$ & $7.29(6.29)$ & 0457 & $0.01^{\mathrm{d}}$ \\
\hline BAI (score) & $9.57(11.11)$ & $9.93(6.92)$ & 0.986 & $0.00^{\mathrm{d}}$ \\
\hline MSNQ (score) & $12.54(9.44)$ & $16.79(11.18)$ & 0.122 & $0.06^{\mathrm{d}}$ \\
\hline NART (score) & $117.38(8.04)$ & $112.57(9.94)$ & 0.302 & $0.03^{\mathrm{d}}$ \\
\hline RAVLT 1-5 (score) & $52.65(9.44)$ & $52.14(9.22)$ & 0.377 & $0.02^{\mathrm{d}}$ \\
\hline RAVLT delayed (score) & $10.73(2.96)$ & $10.50(2.35)$ & 0.453 & $0.01^{\mathrm{d}}$ \\
\hline BVMT-R 1-3 (score) & $27.00(4.49)$ & $25.07(5.39)$ & 0.385 & $0.02^{\mathrm{d}}$ \\
\hline BVMT-R delayed (score) & $10.56(1.29)$ & $9.50(2.07)$ & 0.084 & $0.07^{\mathrm{d}}$ \\
\hline SDMT (score) & $58.00(10.64)$ & $51.21(8.69)$ & 0.034 & $0.11^{\mathrm{d}}$ \\
\hline PASAT (score) & $45.78(10.99)$ & $42.21(12.19)$ & 0.814 & $0.00^{\mathrm{d}}$ \\
\hline Digit span forward (score) & $8.97(2.15)$ & $9.00(1.62)$ & 0.837 & $0.00^{\mathrm{d}}$ \\
\hline Digit span backward (score) & $6.95(2.47)$ & $6.86(2.28)$ & 0.945 & $0.00^{\mathrm{d}}$ \\
\hline TMT A (time in s) & $31.49(9.81)$ & $33.07(7.65)$ & 0.072 & $0.08^{\mathrm{d}}$ \\
\hline TMT B (time in s) & $66.00(22.03)$ & $85.57(29.04)$ & 0.015 & $0.14^{\mathrm{d}}$ \\
\hline COWAT (score) & $41.43(10.73)$ & $33.79(11.76)$ & 0.212 & $0.04^{\mathrm{d}}$ \\
\hline JLO (score) & $26.11(3.58)$ & $24.50(6.12)$ & 0.399 & $0.02^{\mathrm{d}}$ \\
\hline CFT animals (score) & $25.6(6.69)$ & $21.36(4.53)$ & 0.162 & $0.05^{\mathrm{d}}$ \\
\hline CFT shopping (score) & $27.08(8.15)$ & $21.50(4.59)$ & 0.067 & $0.08^{\mathrm{d}}$ \\
\hline \multicolumn{5}{|l|}{ MRI volumetric characteristics ${ }^{\mathrm{c}}$} \\
\hline $\mathrm{nBP}\left(\right.$ volume, $\left.\mathrm{cm}^{3}\right)$ & $1469.09(60.45)$ & $1453.77(95.56)$ & 0.263 & $0.03^{\mathrm{d}}$ \\
\hline nWM (volume, $\mathrm{cm}^{3}$ ) & $682.82(33.44)$ & $664.78(47.11)$ & 0.044 & $0.09^{\mathrm{d}}$ \\
\hline nGM (volume, $\mathrm{cm}^{3}$ ) & $786.27(51.01)$ & $788.99(61.01)$ & 0.905 & $0.00^{\mathrm{d}}$ \\
\hline npGM (volume, $\mathrm{cm}^{3}$ ) & $616.29(40.26)$ & $623.10(48.76)$ & 0.861 & $0.00^{\mathrm{d}}$ \\
\hline Amygdala left (volume, $\mathrm{mm}^{3}$ ) & $1442.17(243.16)$ & $1383.28(220.94)$ & 0.393 & $0.02^{\mathrm{d}}$ \\
\hline Amygdala right (volume, $\mathrm{mm}^{3}$ ) & $1516.93(275.13)$ & $1474.82(264.69)$ & 0.885 & $0.00^{\mathrm{d}}$ \\
\hline Caudate nucleus left (volume, $\mathrm{mm}^{3}$ ) & $3410.73(477.37)$ & $3473.19(634.03)$ & 0.949 & $0.00^{\mathrm{d}}$ \\
\hline Caudate nucleus right (volume, $\mathrm{mm}^{3}$ ) & $3609.67(563.63)$ & $3559.44(589.87)$ & 0.437 & $0.01^{\mathrm{d}}$ \\
\hline Hippocampus left (volume, $\mathrm{mm}^{3}$ ) & $3886.77(565.02)$ & $3758.08(645.75)$ & 0.166 & $0.04^{\mathrm{d}}$ \\
\hline Hippocampus right (volume, $\mathrm{mm}^{3}$ ) & $3951.74(533.41)$ & $3876.73(652.92)$ & 0.514 & $0.01^{\mathrm{d}}$ \\
\hline Globus pallidus left (volume, $\mathrm{mm}^{3}$ ) & $1818.11(219.10)$ & $1812.95(269.69)$ & 0.701 & $0.00^{\mathrm{d}}$ \\
\hline Globus pallidus right (volume, $\mathrm{mm}^{3}$ ) & $1862.36(225.97)$ & $1832.45(278.90)$ & 0.461 & $0.01^{\mathrm{d}}$ \\
\hline Putamen left (volume, $\mathrm{mm}^{3}$ ) & $4988.25(577.08)$ & $5226.59(760.08)$ & 0.335 & $0.02^{\mathrm{d}}$ \\
\hline Putamen right (volume, $\mathrm{mm}^{3}$ ) & $5044.01(607.51)$ & $5143.49(844.39)$ & 0.873 & $0.00^{\mathrm{d}}$ \\
\hline Thalamus left (volume, $\mathrm{mm}^{3}$ ) & $8070.67(856.52)$ & $7807.73(1075.74)$ & 0.204 & $0.04^{\mathrm{d}}$ \\
\hline Thalamus right (volume, $\mathrm{mm}^{3}$ ) & $7864.07(897.40)$ & $7549.57(1072.91)$ & 0.198 & $0.04^{\mathrm{d}}$ \\
\hline \multicolumn{5}{|c|}{ Global and regional MRI lesion load characteristics ${ }^{c}$} \\
\hline Total lesion load (volume, $\mathrm{mm}^{3}$ ) & $2695.92(4302.49)$ & $5126.79(7303.38)$ & 0.110 & $0.06^{\mathrm{d}}$ \\
\hline Caudate nucleus left (volume, $\mathrm{mm}^{3}$ ) & $155.54(180.42)$ & $137.86(222.03)$ & 0.855 & $0.00^{\mathrm{d}}$ \\
\hline Caudate nucleus right (volume, $\mathrm{mm}^{3}$ ) & $135.41(182.62)$ & $154.00(193.34)$ & 0.380 & $0.02^{\mathrm{d}}$ \\
\hline Cerebellum left (volume, $\mathrm{mm}^{3}$ ) & $6.73(24.97)$ & $22.14(53.20)$ & 0.226 & $0.03^{\mathrm{d}}$ \\
\hline Cerebellum right (volume, $\mathrm{mm}^{3}$ ) & $11.27(32.72)$ & $4.79(10.36)$ & 0.634 & $0.01^{\mathrm{d}}$ \\
\hline Frontal lobe left (volume, $\mathrm{mm}^{3}$ ) & $57.41(134.99)$ & $230.43(444.49)$ & 0.020 & $0.11^{\mathrm{d}}$ \\
\hline Frontal lobe right (volume, $\mathrm{mm}^{3}$ ) & $54.68(78.08)$ & $278.86(447.65)$ & 0.006 & $0.16^{\mathrm{d}}$ \\
\hline
\end{tabular}


Table 4 (continued)

\begin{tabular}{|c|c|c|c|c|}
\hline & Normal navigators $(n=37)$ & $\begin{array}{l}\text { Less accurate navigators } \\
(n=14)\end{array}$ & $p$ values & Effect sizes \\
\hline Insula left (volume, $\mathrm{mm}^{3}$ ) & $2.14(5.81)$ & $5.64(17.95)$ & 0.489 & $0.01^{\mathrm{d}}$ \\
\hline Insula right (volume, $\mathrm{mm}^{3}$ ) & $5.14(11.53)$ & $42.86(147.37)$ & 0.167 & $0.04^{\mathrm{d}}$ \\
\hline Occipital lobe left (volume, $\mathrm{mm}^{3}$ ) & $257.27(356.33)$ & $286.71(544.16)$ & 0.576 & $0.01^{\mathrm{d}}$ \\
\hline Occipital lobe right (volume, $\mathrm{mm}^{3}$ ) & $114.95(149.56)$ & $267.86(443.10)$ & 0.093 & $0.06^{\mathrm{d}}$ \\
\hline Parietal lobe left (volume, $\mathrm{mm}^{3}$ ) & $194.51(373.74)$ & $323.86(590.24)$ & 0.286 & $0.03^{\mathrm{d}}$ \\
\hline Parietal lobe right (volume, $\mathrm{mm}^{3}$ ) & $190.51(353.39)$ & $507.43(868.63)$ & 0.049 & $0.08^{\mathrm{d}}$ \\
\hline Putamen left (volume, $\mathrm{mm}^{3}$ ) & $1.92(6.00)$ & $5.79(20.25)$ & 0.454 & $0.01^{\mathrm{d}}$ \\
\hline Putamen right (volume, $\mathrm{mm}^{3}$ ) & $1.73(4.32)$ & $0.64(1.91)$ & 0.294 & $0.02^{\mathrm{d}}$ \\
\hline Temporal lobe left (volume, $\mathrm{mm}^{3}$ ) & $116.35(252.58)$ & $264.07(500.23)$ & 0.079 & $0.07^{\mathrm{d}}$ \\
\hline Temporal lobe right (volume, $\mathrm{mm}^{3}$ ) & $149.14(349.44)$ & $214.29(368.61)$ & 0.422 & $0.01^{\mathrm{d}}$ \\
\hline Thalamus left (volume, $\mathrm{mm}^{3}$ ) & $5.00(15.13)$ & $0.79(2.67)$ & 0.209 & $0.03^{\mathrm{d}}$ \\
\hline Thalamus right (volume, $\mathrm{mm}^{3}$ ) & $4.03(9.64)$ & $5.43(10.68)$ & 0.678 & $0.00^{\mathrm{d}}$ \\
\hline Periventricular left (volume, $\mathrm{mm}^{3}$ ) & $614.24(1268.93)$ & $846.93(1468.24)$ & 0.488 & $0.01^{\mathrm{d}}$ \\
\hline Periventricular right (volume, $\mathrm{mm}^{3}$ ) & $615.87(1170.86)$ & $1524.14(1957.91)$ & 0.054 & $0.08^{\mathrm{d}}$ \\
\hline
\end{tabular}

Demographic, neuropsychological, and MRI characteristics. The values represent mean (SD) unless indicated otherwise. Values in bold indicate significant differences after Holm-Bonferroni correction for multiple comparisons

EDSS Expanded Disability Status Scale, BDI Beck Depression Inventory, BAI Beck Anxiety Inventory, MSNQ Multiple Sclerosis Neuropsychological Questionnaire, NART National Adult Reading Test, RAVLT Rey Auditory Verbal Learning Test, RAVLT delayed RAVLT delayed recall after 30 min, BVMT-R Brief Visuo-spatial Memory Test Revised, BVMT-R delayed BVMT-R delayed recall after 25 min, SDMT Symbol Digit Modalities Test, PASAT Paced Auditory Serial Addition Test, TMT Trail Making Test, COWAT Controlled Oral Word Association Test, JLO Judgement of Line Orientation, $C F T$ Category Fluency Test, $n B P$ normalized brain parenchymal volume, $n W M$ normalized white matter volume, $n G M$ normalized grey matter volume, $n p G M$ normalized peripheral grey matter volume

${ }^{\text {a}}$ Effect size reported using Cohen's $d$

${ }^{b}$ Effect size reported using Cramér's $V$

${ }^{c}$ Based on the analyses controlled for age, gender and education

${ }^{\mathrm{d}}$ Effect size reported using partial eta-squared

Acknowledgements section should read:

The authors would like to thank Ms. Prribylova and Ms. Jozsova for help with data collection and Dr. Lisy for supervising MRI acquisition. This study was supported by the Grant Agency of the Charles University, Prague (no. 546317); Ministry of Health, Czech Republic-conceptual development of research organization, University Hospital Motol, Prague, Czech Republic (no. 00064203); and InstitutionalSupport of Excellence 2. LF UK (no. 699332).

The original article has been corrected. 